\title{
A Sex Difference in Synaptic Efficacy at the Laryngeal Neuromuscular Junction of Xenopus laevis
}

\author{
Martha L. Tobias, ${ }^{1}$ Darcy B. Kelley, ${ }^{1}$ and Mark Ellisman ${ }^{2}$ \\ ${ }^{1}$ Department of Biological Sciences, Columbia University, New York, New York 10027 and ${ }^{2}$ San Diego Microscopy \\ and Imaging Resource, Department of Neuroscience, UCSD Medical School, La Jolla, California 92093
}

\begin{abstract}
Under physiological conditions, the response of Xenopus laevis laryngeal muscle fibers to nerve stimulation is sexually differentiated; subthreshold potentials are common in males and rare in females. This sex difference in muscle fiber response is correlated with sex differences in vocal behavior. Quantal analyses at male and female laryngeal synapses were performed to determine if there is a sex difference in synaptic strength. Quantal content at laryngeal synapses is significantly higher in females than in males. Values for quantal content in males can be increased by raising extracellular calcium concentration. There is no sex difference in miniature endplate potential amplitude suggesting that $\mathrm{ACh}$ receptor number or properties are not different in the sexes. Sex differences in synaptic strength thus appear presynaptic in origin; transmitter release is less in males. Ultrastructural analyses of the laryngeal motor terminal indicate that there is no sex difference in the length of active zones or in the number of channels per length of active zone. Thus, ultrastructural characteristics of the laryngeal motor terminal do not account for the pronounced sex difference in quantal content.

[Key words: active zones, frog, miniature endplate potentials, quantal content, synaptic strength, vocal behavior]
\end{abstract}

Sex differences in connectivity between neurons and their targets-other neurons or muscle fibers-are thought to underlie sex-typical behaviors. One way connections could differ is in the number of synapses mediating the behavior and there is extensive evidence for sex differences in synaptic density, dendritic extent, axonal branching and cell number (reviewed in Kelley, 1988). Another way connections in the sexes could differ is in the strength of synapses. However, it has been difficult to demonstrate sex differences in synaptic efficacy within the complex CNS circuits which control sex-typical behaviors. To investigate this problem we have been studying a synapse which is both accessible and known to mediate a sexually differentiated behavior-the laryngeal neuromuscular synapse, the final effector for vocal behavior in Xenopus laevis frogs. In this article we demonstrate a marked difference in synaptic strength at the laryngeal neuromuscular junction of males and females and pro-

\footnotetext{
Received May 18, 1994; revised Aug. 23, 1994; accepted Aug. 30, 1994.

We are grateful to $\mathrm{S}$. Kahn, E. Parker, and T. Ruel for excellent technical assistance. This work was supported by NS 23684 (D.B.K.) and by NS 2739 and RR 04050 (M.H.E.).

Correspondence should be addressed Dr. Martha Tobias, Department of Biological Sciences, 909 Fairchild, Columbia University, New York, NY 10027 Copyright 1995 Society for Neuroscience $0270-6474 / 95 / 151660-09 \$ 05.00 / 0$
}

vide evidence for a sex difference in transmitter release from the motor terminal.

The vocal behavior of Xenopus laevis differs markedly in males and females. The male produces a courtship song of rapid, modulated trills to attract and excite females (Picker, 1983; Wetzel and Kelley, 1983). Females cannot produce courtship song (Hannigan and Kelley, 1986; Tobias and Kelley, 1987) but do produce a slower, unmodulated call which is used to repel males (Russell, 1954; Weintraub et al., 1985). Because sound production does not rely on air flow in Xenopus (Yager, 1992), the vocal organ or larynx can be removed from the frog and actual vocalizations produced by stimulating the laryngeal nerve (Tobias and Kelley, 1987). We have identified several laryngeal characteristics, including muscle fiber number and twitch type, that contribute to the male's ability to produce rapid and prolonged trills (Watson and Kelley, 1992; Watson et al., 1993). The pronounced sex difference in the response of muscle fibers to nerve stimulation in isolated larynges suggests that the neuromuscular synapse is another locus for mediating sex differences in vocal behavior (Tobias and Kelley, 1987, 1988). Under physiological conditions, the majority of muscle fibers in male larynges generate subthreshold potentials in response to a single nerve stimulus. Action potentials in these fibers are only produced following repetitive nerve stimulation. In contrast, the majority of muscle fibers in female larynges generate an action potential in response to nerve stimulation, either single or repetitive; no subthreshold potentials are observed in these fibers.

The sex difference in the frequency of subthreshold potentials produced in response to nerve stimulation suggests a sex difference in strength at the laryngeal neuromuscular synapse. To further characterize this sex difference, synaptic efficacy was analyzed by measuring quantal content and miniature endplate potential amplitude at male and female laryngeal neuromuscular synapses. In addition, some ultrastructural characteristics of the motor terminal which could contribute to synaptic strength, active zone length and channel density, were examined.

\section{Materials and Methods}

\section{Experimental preparation}

Adult (greater than 2 years of age) male and female frogs were obtained from Nasco Co. (Ft. Atkinson, WI). At this stage (PM6 of Tobias et al., $1991 \mathrm{~b}$ ) body weights (bw) in males are $>25 \mathrm{gm}$ and laryngeal weights (lw) $>350 \mathrm{mg}$; corresponding values for females are $>50 \mathrm{gm}$ (bw) and $150 \mathrm{mg}(\mathrm{lw})$. Animals were maintained in polycarbonate tanks containing dechlorinated water under a 12:12 (light:dark) cycle and fed frog brittle three times per week.

All experiments were performed on isolated larynges (Tobias and Kelley, 1987). Intracellular recordings were typically obtained from fibers beneath the muscle surface and the endplate could not be visualized 
during recordings. To ensure that cable properties and distance to the endplate did not account for differences in quantal measurements, input resistance and miniature endplate potential (mepp) rise time were measured. Acetylcholinesterase histochemistry and bungarotoxin staining revealed an intense band of endplates located medially within the larynx (data not shown); the electrode was directed toward this region and with experience synaptic potentials could be recorded readily suggesting proximity to a synapse.

Quantal content measurements were obtained in low calcium, high magnesium saline to prevent action potential production in female fibers. Mepp amplitude was measured in high calcium or sucrose saline to increase mepp frequency. Because of experimental difficulties imposed by changing saline, quantal content and mepps were not obtained from the same muscle fiber. Instead, populations of fibers from each sex were analyzed and compared using two-tailed, unpaired $t$ tests $(N$ $=$ number of synapses).

\section{Electrophysiology}

Quantal content. Frogs were deeply anesthetized by immersion in $0.1 \%$ MS22.2 (ethyl $m$ amino benzoate methane sulfonic acid, Aldrich). I arynges were removed and bathed in normal frog saline $(116 \mathrm{mM} \mathrm{NaCl}$, $2 \mathrm{mM} \mathrm{KCl}, 2.5 \mathrm{~mm} \mathrm{CaCl}_{2}, 3.0 \mathrm{mM} \mathrm{MgCl}, 27.7 \mathrm{~mm}$ dextrose, $4 \mathrm{~mm}$ HEPES buffer). The laryngeal nerve was stimulated and laryngeal muscle contractions evoked in female larynges bathed in normal saline. The $\mathrm{Ca}^{2+}$ ion concentration was then lowered and the $\mathrm{Mg}^{2+}$ ion concentration was raised until laryngeal muscle contractions were no longer visible. This concentration ( $105 \mathrm{mM} \mathrm{NaCl}, 2 \mathrm{mM} \mathrm{KCl}, 0.5 \mathrm{mM} \mathrm{CaCl}_{2}$, and $5 \mathrm{mM} \mathrm{MgCl}$ ) was then used for measurements of quantal content $(m)$ at 29 male and 21 female synapses $(9$ male and 12 female frogs were used). The $\mathrm{NaCl}$ concentration was adjusted to maintain osmolarity in all salines in which $\mathrm{Ca}^{2+}$ or $\mathrm{Mg}^{2+}$ ion concentration was changed. $\mathrm{La}$ ryngeal muscle fibers were impaled with glass microelectrodes, 25-40 M $\Omega$ resistance. A failure analysis (Del Castillo and Katz, 1954) was used to estimate quantal content. The laryngeal nerve was stimulated at $3 \mathrm{sec}$ intervals (see Tobias and Kelley, 1988, for details) and the proportion of postsynaptic potential (psp) failures was determined (number of trials per synapse ranged from 50 to 185). Quantal content was calculated as $\ln$ (total \# of trials/\# of failures). The significance of differences in quantal content at male and female neuromuscular synapses was evaluated with an unpaired, two-lailed $t$ test; all values given are means \pm standard deviations. Quantal content was also determined for 13 male synapses ( 5 male frogs were used) at the ion concentration which just blocks visible muscle contractions in male larynges ( $1.5 \mathrm{~mm}$ $\mathrm{CaCl}_{2}, 4 \mathrm{mM} \mathrm{MgCl}_{2}$ ); these data were compared with measurements obtained from males tested in $0.5 \mathrm{~mm} \mathrm{CaCl} 2,5.0 \mathrm{mM} \mathrm{MgCl}_{2}$ using an unpaired, two-tailed $t$ test. Quantal content for females cannot be determined under these ion conditions because stimulation of the laryngeal nerve produces muscle action potentials.

Mepp amplitude. Mepp frequency is very low under physiological conditions. Measurements of mepp amplitude were thus obtained from preparations in high calcium $(15 \mathrm{~mm})$ or sucrose $(0.25 \mathrm{M})$ saline. At $0.25 \mathrm{M}$ sucrose or $15 \mathrm{~mm}$ calcium, mepp frequency is between 0.3 and $1 \mathrm{~Hz}$ and individual mepps are readily distinguished. Mepp amplitude and input resistance were measured in both saline conditions; mepp rise times were measured in high calcium saline only. A muscle fiber was impaled and any electrical activity recorded. If there was no spontaneous activity after two minutes, the electrode was moved to a new position. Mepp amplitude, measured to the nearest $0.5 \mathrm{mV}$ directly from the storage oscilloscope, was recorded at 25 male and 17 female laryngeal neuromuscular junctions from 14 male and 12 female frogs. The number of mepps recorded at each synapse ranged from 20 to 70 ; means for each synapse were compared in males and females using an unpaired, two-tailed $t$ test ( $N=$ number of synapses). The frequency of mepp amplitude classes in $0.5 \mathrm{mV}$ intervals was also computed across male and female synapses; a frequency plot was constructed and the mean and standard deviation for each amplitude class compared.

Mepp rise time. To ensure that there was no consistent sex difference in the distance between the endplate and the recording electrode, mepp rise time was measured in a subset of fibers used for measurements of mepp amplitude. Spontaneous activity from these fibers (five male and five female) recorded in $15 \mathrm{~mm}$ calcium were stored on tape (Vetter, model B) and subsequently transferred to a computer for analysis (Macintosh IIcx, SUPERSCOPE II software). The time from baseline to peak amplitude was measured for 10 mepps from each synapse (100 mepps total). An one-way ANOVA with repeated measures was used to de- termine the effect of sex. A correlation coefficient between mepp amplitude and mepp rise time was also determined.

Input resistance. Because a fiber's input resistance can influence both the detection of evoked potentials and measurements of mepp amplitude, measurements of input resistance were obtained in a subset of fibers from which quantal content (10 females, 13 males) or mepp amplitude ( 6 females, 12 males) had also been obtained. The electrode resistance was measured in saline prior to impaling a fiber. Once a fiber was impaled, current pulses, $5 \mathrm{msec}$ in duration, were passed through the electrode and the resulting voltage change measured directly from a storage oscilloscope. Each fiber was stimulated with five current pulses; range of stimulus strengths for all fibers varied from -1 .0nA to $+0.7 \mathrm{nA}$. The slope of the current/voltage curve was used to calculate total resistance; input resistance was calculated by subtracting the electrode resistance from the total resistance. An unpaired. two-tailed $t$ test was used to determine whether input resistance differs in the sexes $(N$ $=$ number of fibers).

\section{Active zone morphology}

Freeze fracture microscopy was used to examine release sites in the presynaptic terminal of male and female laryngeal neuromuscular junctions. Nine male and nine female frogs were perfused with normal frog saline followed by 2 or $4 \%$ paraformaldehyde $/ 2 \%$ glutaraldehyde in 0.1 M phosphate buffered saline. Laryngeal muscle was dissected into small bundles of fibers, slowly equilibrated in $30 \%$ glycerol, placed on brassmesh specimen supports and quickly frozen in liquid Freon 12. Tissue was fractured at $-150^{\circ} \mathrm{C}$ and was replicated at $-103^{\circ} \mathrm{C}$ on a freeze etch device (Balzer). Replicas were prepared with a platinum-carbon gun and examined with an electron microscope (JEOL 100CX). Stereo pairs were produced by tilting sections at angles ranging from $+10^{\circ}$ to $-10^{\circ}$ and were used to insure that only flat regions of membrane were used for analysis of active zone length ( 80 female, 78 male) and density of intramembranous particles ( 18 female, 11 male). Photographs of each fractured endplate were taken and the active zones traced and measured with a bit pad (Sigma Scan, Jandel Scientific). An active zone was defined by the presence of two apposed rows of intramembranous particles (Dreyer et al., 1973; see Fig. $4 B, D$ ); three measurements were taken of each active zone and the mean used to define length. In laryngeal terminals, active zones occur in linear arrays that are frequently interrupted by stretches of membrane without intramembranous particles. If rows of intramembranous particles were interrupted by $>50 \mathrm{~nm}$, these were considered separate active zones (Pumplin, 1983). The number of intramembranous particles associated with an active zone was counted in preparations in which the particles were clearly visible; the number of particles in one row on one side of an active zone was determined. Unpaired, two-tailed $t$ tests were used to evaluate the significance of differences in these measurements between males and females $(N=$ number of active zones).

\section{Results and Specific Discussion}

\section{Sex differences in synaptic strength}

In physiological saline (containing $2.5 \mathrm{mM} \mathrm{CaCl}_{2}, 3.0 \mathrm{~mm}$ $\mathrm{MgCl}_{2}$ ), nerve evoked responses are suprathreshold in female and subthreshold in male laryngeal muscle fibers (Tobias and Kelley, 1988). In order to compare quantal content in male and female laryngeal muscle fibers under the same ion conditions, synaptic transmission was depressed by lowering the calcium and raising the magnesium ion concentrations until visible muscle contractions were prevented in both sexes. While determining these ion concentrations, we noted that considerably more stringent ion conditions (lower calcium, higher magnesium) were needed to prevent muscle contractions at female $(0.5 \mathrm{~mm}$ $\mathrm{CaCl}_{2}, 5.0 \mathrm{mM} \mathrm{MgCl}_{2}$ ) than at male $\left(1.5 \mathrm{mM} \mathrm{CaCl}_{2}, 4.0 \mathrm{~mm}\right.$ $\mathrm{MgCl}_{2}$ ) synapses. Intracellular recordings revealed that action potentials were never produced under ion conditions that prevent visible muscle contractions. These observations provide further evidence that there is a sex difference in synaptic strength and suggest that this difference is calcium dependent.

Quantal content $(m)$, the mean number of quanta released in response to nerve depolarization, was estimated using an anal- 

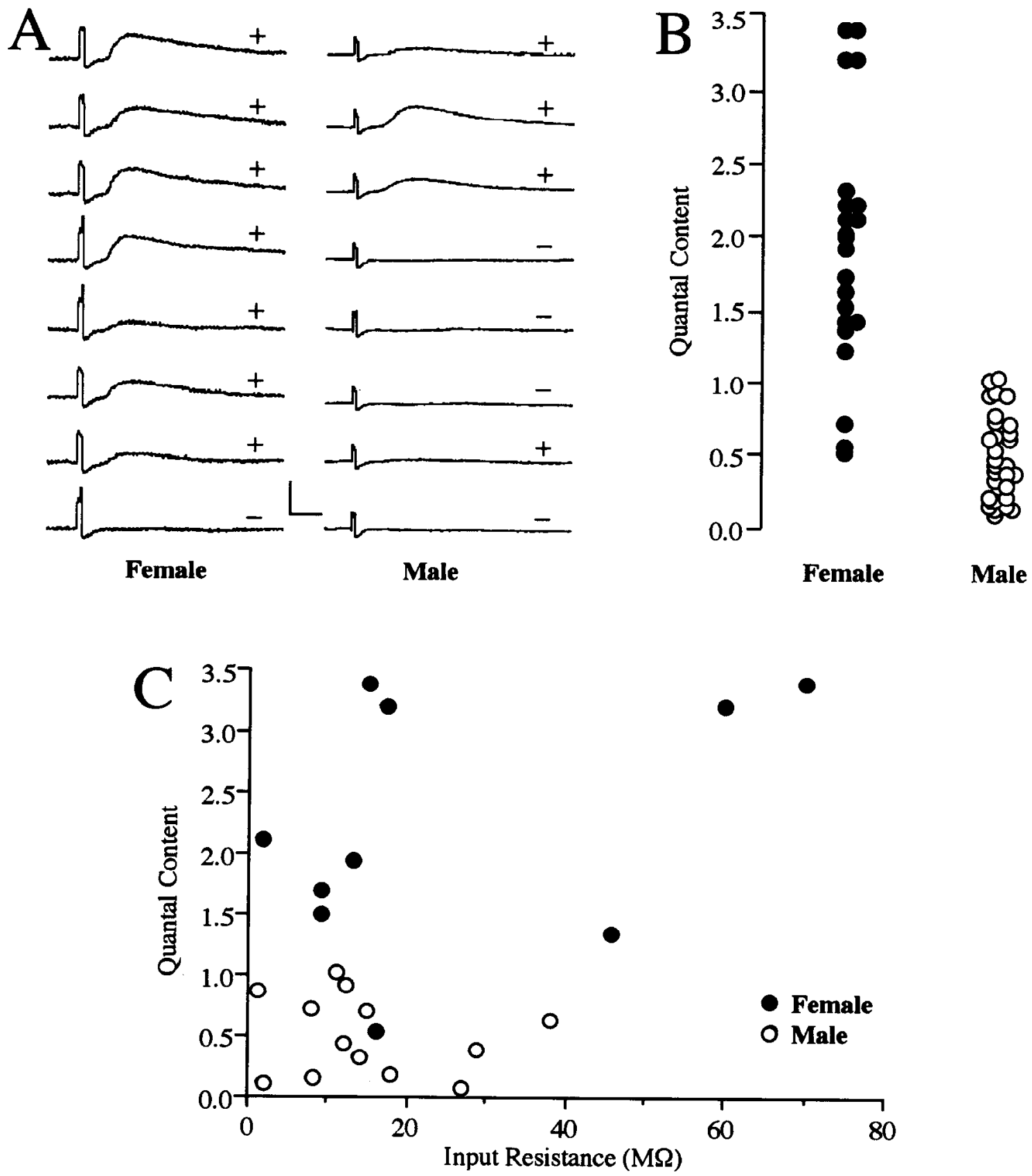

Figure 1. Quantal content is higher at female than at male laryngeal synapses. A, Muscle fiber responses, postsynaptic potentials and failures, at a female and a male laryngeal neuromuscular synapse. The number of failures is larger and the end-plate potential is smaller in males. Calibration: $10 \mathrm{mV}, 5 \mathrm{msec}$. B, Values for quantal content [ln (\# of trials/\# of failures)] are shown for 21 female and 29 male synapses. $C$, Quantal content as a function of input resistance for a subset of the fibers shown in $B$, There is no sex difference in input resistance.

ysis of failures. The ion concentration which prevents nerve evoked muscle action potentials in female larynx $(0.5 \mathrm{mM} \mathrm{CaCl}$, $5.0 \mathrm{mM} \mathrm{MgCl}_{2}$ ) was used to measure quantal content at male and female symapses. Examples of muscle responses to nerve stimulation are illustrated in Figure $1 A$. While the amplitude of the evoked psp varies in both sexes, psp amplitude is typically smaller in males. Failures are readily distinguished from small responses. The proportion of failures is greater at male than at female synapses. Quantal content values (Fig. 1B) are consistently higher at female than at male laryngeal synapses (female mean $m=1.90 \pm 0.8$, male mean $m=0.45 \pm 0.3, p<.0001$ ).

Because quantal content might be influenced by fiber input resistance, we obtained both measurements in a subset of fibers (Fig. 1C). Input resistance measured from 10 female and 13 male fibers did not reveal a significant sex difference (female mean $=25.7 \pm 23.8$, male mean $=15.2 \pm 10.3 ; p=0.15$ ). For these same fibers, quantal content is significantly different in the sexes $(p<0.0001$; female mean $=2.3 \pm 1.0$, male mean $=0.4 \pm 0.4$ ). Male and female fibers with similar input resistances had markedly different quantal contents while fibers with similar quantal contents, that is, from the same sex, had markedly different input resistances. Thus, it is unlikely that differences in input resistance contribute to the observed sex difference in quantal content. 


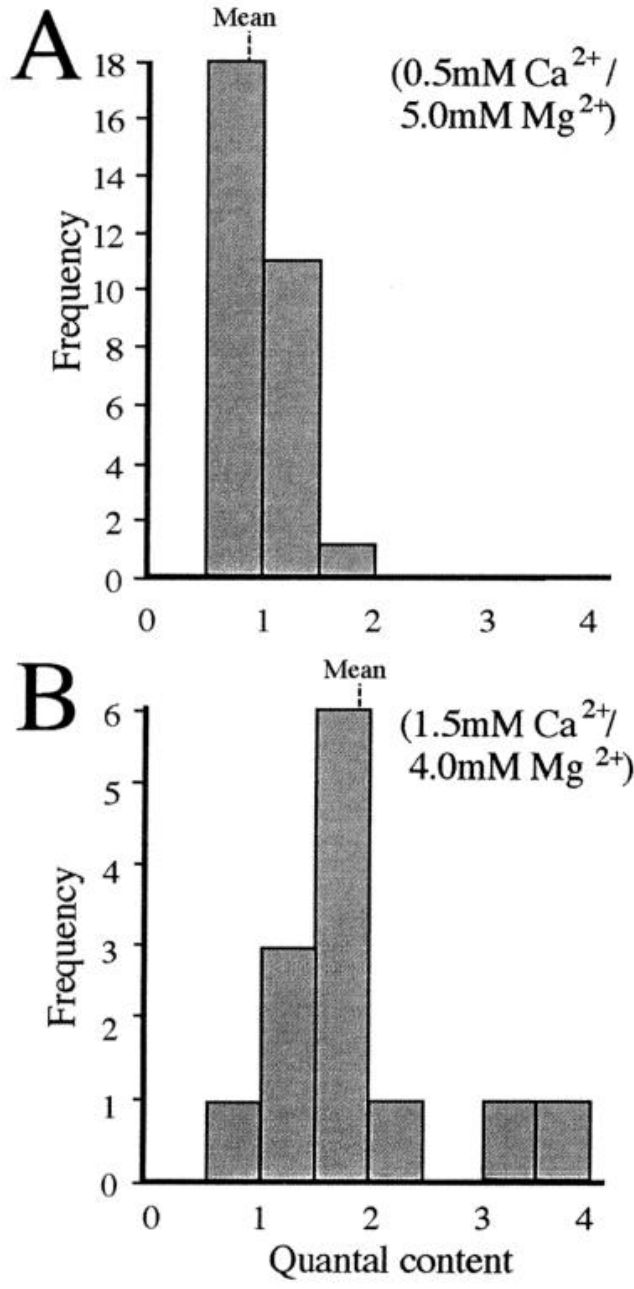

Figure 2. Quantal content in male larynx is dependent on calcium and magnesium ion concentrations. $A$, Distribution of quantal content values obtained in $0.5 \mathrm{mM} \mathrm{Ca}^{2+} / 5.0 \mathrm{mM} \mathrm{Mg}{ }^{2+}$ saline (from Fig. 1). B, Distribution of quantal content values obtained in $1.5 \mathrm{~mm} \mathrm{Ca}^{2+} / 4.0 \mathrm{~mm} \mathrm{Mg} \mathrm{Mg}^{2+}$ (the ion concentration required to block action potentials and contractions in male laryngeal muscle). Quantal content is significantly increased in higher $\mathrm{Ca}^{2+} /$ lower $\mathrm{Mg}^{2+}$ saline.

Measurements of $m$ were also obtained for male laryngeal synapses at the ion concentration which just prevents action potentials in this sex $\left(1.5 \mathrm{mM} \mathrm{CaCl}_{2}, 4.0 \mathrm{mM} \mathrm{MgCl}\right.$ ) and compared (Fig. $2 B$ ) to values obtained at the ion concentration which just blocks action potentials in females $\left(0.5 \mathrm{mM} \mathrm{CaCl}_{2}, 5.0 \mathrm{~mm}\right.$ $\mathrm{MgCl}_{2}$; Fig. 2A). Quantal content could not be measured in females under the former ion conditions because muscle responses are suprathreshold. Raising the calcium and lowering the magnesium concentration decreases the number of synaptic failures and thus significantly increases quantal content at male laryngeal neuromuscular junctions ( $1.7 \pm 0.6$ vs $0.45 \pm 0.3 ; p<0.0005)$. Values for quantal content are more broadly distributed under less stringent ion conditions revealing an underlying variability in synaptic strength not apparent at lower calcium concentrations.

We conclude that quantal content is significantly less at male than at female neuromuscular synapses when measured under the same ion conditions. Since females generate action potentials and males generate subthreshold potentials in physiological sa- line (Tobias and Kelley, 1988), we presume that quantal content is also lower in males under physiological conditions.

The sex difference in quantal content suggests that transmitter release from the presynaptic terminal contributes to sex differences in synaptic efficacy. However, these results do not exclude an alternative possibility, that the more frequent synaptic failures of males reflect a sex difference in postsynaptic properties such as number or conductance properties of acetylcholine receptors. A sex difference in such postsynaptic receptor characteristics should be accompanied by a sex difference in the amplitude of postsynaptic responses to spontaneously secreted transmitter or miniature endplate potentials. Neither the size of individual mepps (Fig. $3 A$ ), nor the distribution of mepp amplitudes at individual synapses (Fig. $3 B$ ), differed in males and females. Mepp amplitude is normally distributed although the mean and range of amplitudes varies between synapses (Fig. $3 B$ ). Mean mepp amplitude at each synapse is shown (Fig. $3 C$ ); there is no significant sex difference in mean mepp amplitude (males $=1.1$ $\pm 0.6 \mathrm{mV}$, females $=1.8 \pm 1.2 \mathrm{mV} ; p>0.1$ ). Because input resistance can influence measurements of mepp amplitude, input resistance was measured in 12 male and 6 female fibers in which mepp amplitudes were also recorded (Fig. 3D). There is no sex difference in input resistance of these fibers (male $=21.8 \pm$ 12 , female $=20.8 \pm 9 ; p>0.9$ ). Mepp amplitude is similar in fibers with markedly different input resistances for both sexes. The frequency distribution of mepp amplitudes averaged across male and female synapses was analyzed (Fig. $3 E$ ). The relative proportion of mepp amplitudes does not differ in males and females; the mean frequency of each mepp amplitude class falls within $1 \mathrm{SD}$ of the mean for the other sex.

Mepp amplitude is also influenced by the distance between the synapse and the recording electrode; the further from the synapse the smaller the amplitude and the longer the rise time. Thus, mepp rise time was measured to insure that there was no consistent sex difference in recording distance from the synapse. The mean rise time for an experiment varies in both sexes; the range of rise times for females is from $1.0 \pm 0.2 \mathrm{msec}$ to 1.7 $\pm 0.1 \mathrm{msec}$ and for males is from $1.2 \pm 0.5 \mathrm{msec}$ to $2.1 \pm$ $0.7 \mathrm{msec}$. An ANOVA indicates that there is no significant difference in mean rise time between the sexes (female mean = $1.4 \pm 0.3$, male mean $=1.6 \pm 0.4 ; p>0.3)$. The correlation between rise time and amplitude is 0.2 for both sexes suggesting that the variability in recording distance does not strongly affect mepp amplitude measurements. Taken together, these data indicate that there is no measurable sex difference in mepp amplitude at laryngeal synapses. Thus, sex differences in postsynaptic receptor characteristics do not account for sex differences in quantal content.

\section{Active zone morphology at male and female laryngeal motor terminals}

Our analyses suggest that sex differences in synaptic efficacy result from sex differences in transmitter release at the motor terminal. Transmitter release occurs via vesicle fusion at specialized presynaptic sites termed active zones. The active zone is closely associated with intramembranous particles believed to be the calcium channels required for vesicle fusion (Pumplin et al., 1981). Longer active zones could influence transmitter release by providing more sites for vesicle fusion. The density of putative calcium channels could influence intracellular calcium levels and thus affect transmitter release. We have therefore examined presynaptic terminals of laryngeal motor neurons for 

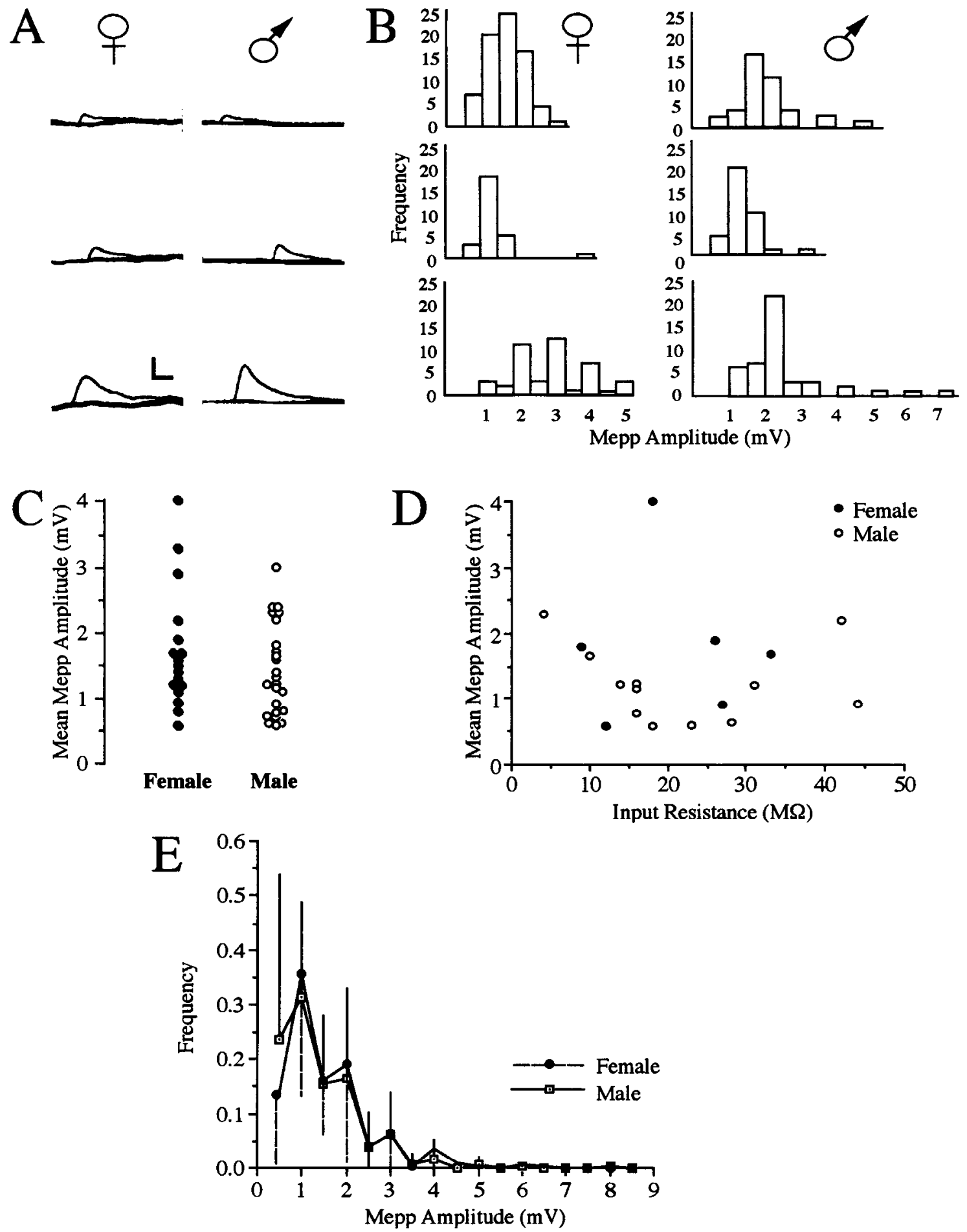

Figure 3. Miniature end-plate potential amplitudes from female and male laryngeal synapses are not different. $A$, Representative mepps recorded at a female and a male synapse. Calibration: $5 \mathrm{mV}, 5 \mathrm{msec}$. $B$, The distribution of mepp amplitudes recorded from three female and three male synapses. Mepp amplitude is normally distributed and similar in the sexes. $C$, Comparison of mean mepp amplitudes at all male and female synapses. There is no significant sex difference in mean mepp amplitude. $D$, Input resistance vs mepp amplitude in a subset of fibers shown in $C$; there is no significant difference in input resistance. $E$, Frequency distribution of mepp amplitudes from male and female laryngeal synapses. The mean and SD of values for each amplitude class were obtained separately for males and females; values for males and females fall within 1 SD of each other at all amplitudes and thus did not differ significantly.

possible sex differences in active zone length and density of intramembranous particles.

The active zone and its associated channels can be visualized in freeze fracture replicas of male and female motor terminals
(Fig. 4). Under favorable circumstances, sites of vesicle fusion at the active zone are observed (Fig. $4 B$ ). Active zones are frequently organized in linear arrays parallel to the transverse axis of the motor terminal. Because the plane of fracture is random, 

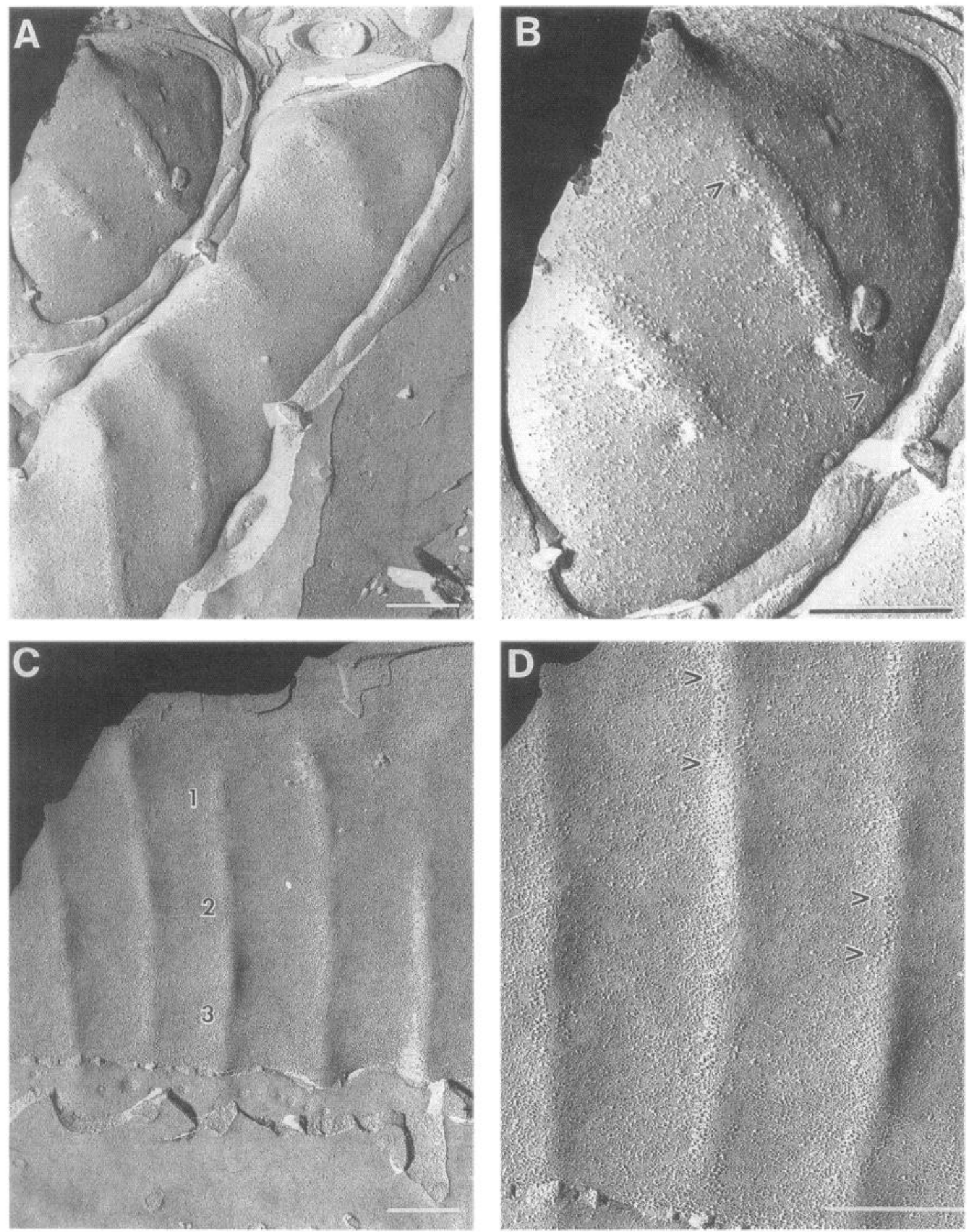

Figure 4. Freeze fracture images from a male and a female laryngeal motor terminal. Lower magnification micrographs of active zones in the female (A) and male $(C)$; active zones are arranged perpendicular to the length of the terminal. Active zones are arranged in linear arrays $(C$, $1-$ 3 ) opposite a junctional fold. Higher magnification micrographs of the female $(B)$ and the male $(D)$ fractures. At this magnification the double rows of intramembranous particles (arrowheads) are clearly visible. In some fractures $(B)$, larger particles represent sites of synaptic vesicle fusion with the membrane. Scale bars, $0.5 \mu \mathrm{m}$.

replicas more frequently included only a portion of the motor terminal (Fig. $4 C$ ) rather than the entire width (Fig. 4A). The distribution of lengths for 78 male and 80 female active zone is shown (Fig. 5). Active zone length is highly variable although most active zones are short $($ mean $=0.26 \mu \mathrm{m})$. Longer active zones $(>0.3 \mu \mathrm{m})$ are more frequently found in females (Figs.
$4 B, 5)$ but there is no sex difference in mean active zone length (females $=0.28 \pm 0.15 \mu \mathrm{m}$; males $=0.24 \pm 0.12 \mu \mathrm{m} ; p>$ 0.2 ). The density of intramembranous particles at 11 male and 18 female active zones was determined by counting the number in one row of the doublet and dividing by the active zone length. Particle number $/ \mu \mathrm{m}$ active zone is not significantly different be- 

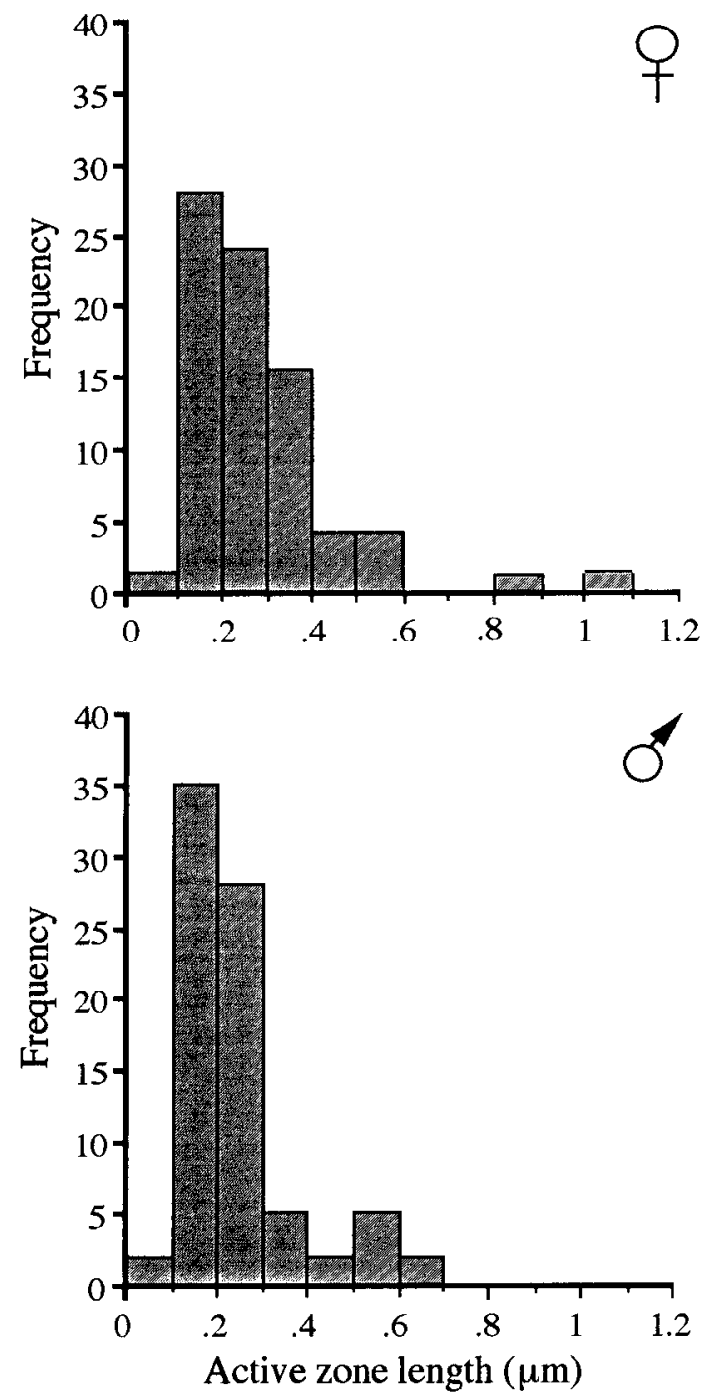

Figure 5. Frequency distribution of active zone lengths in male and female laryngeal motor terminals. There is no significant sex difference in mean active zone length. The frequency of longer active zones is slightly greater in female terminals.

tween the sexes $($ female $=56.5 \pm 15.8$, male $=58.3 \pm 8.3 ; p$ $>0.7$ ). We conclude that neither active zone length nor the density of channels associated with the active zone is sexually dimorphic at the laryngeal neuromuscular synapse and thus neither contributes to the observed sex difference in quantal content.

\section{Discussion}

Sex differences in synaptic efficacy are controlled presynaptically

We have demonstrated a marked sex difference in the strength of synapses formed by laryngeal motor neurons on their target muscle fibers. This sex difference is apparent at physiological calcium and magnesium levels and accounts for the high frequency of subthreshold synaptic potentials at male laryngeal synapses (Tobias and Kelley, 1988). The data presented here strongly suggest that sex differences in synaptic strength are presynaptic in origin. Quantal content, the mean number of quanta released in response to depolarization of the presynaptic membrane, is less in males than in females. Transmitter release is calcium dependent and quantal content at the laryngeal synapse is affected by extracellular calcium. Further, there is no sex difference in the amplitude of miniature endplate potentials; a difference would be expected if the conductance or number of ACh-activated channels differed in males and females. Although there is clearly a presynaptic contribution to sex differences in synaptic strength at the laryngeal neuromuscular junction, we do not rule out additional postsynaptic components. A postsynaptic mechanism has been postulated to account for the sex difference in electric organ discharge exhibited by the weakly electric fish, Sternopygus (Zakon, 1993). Discharge frequency is regulated by electrocyte action potential duration; the underlying mechanism appears to be a sex difference in sodium channel kinetics (Ferrari and Zakon, 1993).

The quantal analyses suggest that the underlying mechanisms for a sex difference in synaptic strength involve the regulation of transmitter release. One possible site for regulation could be the locus of synaptic vesicle fusion with the membrane, the active zone, in which case we might expect to observe a morphological difference in presynaptic terminals on male and female laryngeal muscle. Reduced transmitter release seen during development or produced by nerve damage is associated with shorter active zone segments (Ko, 1981, 1984, 1985). Changes in active zone length have also becn shown to accompany simple learned behaviors; active zone length is increased following long-term sensitization and decreased following long-term habituation (Bailey and Chen, 1989). In Rana pipiens, synapses on cutaneous pectoris have been shown to be more effective than those on cutaneous dorsi (Banner and Herrera, 1986); at the less efficacious synapse, active zone length per unit terminal is shorter (Propst et al., 1986). In contrast to these studies, however, our ultrastructural analyses in Xenopus larynx revealed no sex differences in active zone length.

Another way in which sex differences in transmitter release might be produced is via regulation of the number of calcium and/or calcium dependent potassium channels in the presynaptic terminal. The calcium channels at the Xenopus laryngeal synapse are probably the N-type specific to neurons (Tsien et al., 1988 ) as these are present at synapses on other Xenopus muscles (Cohen et al., 1991) and have been shown to influence transmitter release at Rana cutaneous pectoris synapses (Robitaille et al., 1990). Calcium dependent potassium channels also affect transmitter release (Robitaille and Charlton, 1992) and have been shown to colocalize with calcium channels at the frog neuromuscular junction (Robitaille et al., 1993). Our data suggest that the number of channels/ $\mu \mathrm{m}$ active zone is similar in the sexes. Studies on cutaneous pectoris and dorsi also revealed no difference in active zone intramembranous particle density between more and less efficacious synapses (Propst et al., 1986). Because freeze fracture does not distinguish between calcium and potassium channels, we cannot exclude the possibility that differences in the relative numbers of channel types accompany differences in synaptic efficacy. Additional points of regulation which could produce sex differences in transmitter release include calcium channel kinetics and characteristics of proteins involved in synaptic vesicle mobilization and fusion (reviewed in Greengard et al., 1993; Popov and Poo, 1993).

\section{Organizing a sexually dimorphic circuitry}

The presence of certain profound structural and functional sex differences in the CNS is strongly correlated with sexually differentiated features of behavior. For the most part, research has 
concentrated on experimental systems in which males perform behaviors that females do not. Certain brain nuclei implicated in the control of such behaviors are larger in volume, often with more cells and enriched synaptic neuropil (reviewed in Kelley, 1988; Breedlove, 1992). In extreme cases, such as the motor nucleus (SNB) innervating the levator ani/bulbocavernosus muscles of male rats or the telencephalic nucleus $\mathrm{X}$ of song birds, a structure that is robust in the male is barely detectable in the female. The origin of the dramatic sex difference in the SNB probably results from androgen regulated prevention of ontogenetic cell death in males (reviewed in Forger et al., 1992).

The sex differences that characterize the vocal system of $X$. laevis are not as extreme as those of the rat levator ani/bulbocavernosus system. For example, while the number of laryngeal motor neurons and muscle fibers is greater in males (Kelley and Dennison, 1990; Marin et al., 1990), in X. laevis both sexes maintain the vocal organ into adulthood and both use laryngeal motor neurons and muscles to effect social communication. The way the vocal neuromuscular system functions in adult males and females is, however, quite different. A major goal of this study was to determine the cellular property which accounts for the sex difference in synaptic strength. The present results suggest that the different functional characteristics of male and female laryngeal synapses are achieved via regulation of transmitter release. The next goal is to determine how transmitter release is hormonally regulated. We have shown that cell numbers in the laryngeal neuromuscular system are controlled by androgen secretion during late tadpole and early postmetamorphic development (Marin et al., 1990; Robertson et al., 1994). Androgenic steroids are also good candidates for control of synaptic efficacy in adults and have been shown to reduce synaplic efficacy at another androgen-sensitive synapse-on "clasper" muscles - in Xenopus laevis (Nagaya and Herrera, 1991). In the levator ani of developing male rats, results of experiments using muscle twitch tension in depleted calcium saline to estimate synaptic efficacy suggest that androgen lowers synaptic strength at some developmental stages (Jordan et al., 1992).

\section{Weak vocal synapses are required for attractive songs}

The songs used by male Xenopus laevis to attract females are amplitude modulated trills (Picker, 1983; Wetzel and Kelley, 1983). To determine whether amplitude modulation is essential for the attractive quality of the call, we presented females with a choice between normal, modulated songs and those in which amplitude modulation had been removed from the fast trill portion; females clearly preferred modulated to unmodulated songs (Tobias et al., 1991a). The gradually increasing loudness of the fast trill accompanies the progressive recruitment of laryngeal muscle fibers that occurs in response to repetitive nerve stimulation (Tobias and Kelley, 1987). We have postulated that fiber recruitment relies on facilitation of weak male neuromuscular synapses (Tobias and Kelley, 1988). Presumably, males with strong vocal synapses could not produce amplitude modulated songs.

The quality of song (i.e., degree of amplitude modulation) could serve as an indication of the reproductive status of the singer and be used by a female for mate choice. The songs of males may even have evolved to suit an auditory preference of females for calls which start softly and become louder, perhaps because such songs are more easily localized. In either case, males producing modulated calls would have a reproductive advantage over those limited to unmodulated forms.

\section{References}

Bailey $\mathrm{CH}$, Chen M (1983) Morphological basis of long-term habituation and sensitization in Aplysia. Science 22:91-93.

Banner L, Herrera AA (1986) Differences in synaptic efficacy at neuromuscular junctions in frog twitch muscles. J Physiol (Lond) 379: 205-215.

Breedlove SM (1992) Sexual dimorphism in the vertebrate nervous system. J Neurosci 12:4133-4142.

Cohen MW, Jones O'l; Angelides KJ (1991) Distribution of $\mathrm{Ca}^{2+}$ channels on frog motor nerve terminals revealed by fluorescent $\omega$-conotoxin. J Neurosci 11:1032-1039.

Del Castillo J, Katz B (1954) Quantal components of the end-plate potential. J Physiol (Lond) 124:560-573.

Dreyer F, Peper K, Akert K, Sandri C, Moor H (1973) Ultrastructure of the "active zone" in the frog neuromuscular junction. Brain Res 62:373-380.

Ferrari M, Zakon H (1993) Conductances contributing to the action potential of Sternopygus electrocytes. J Comp Physiol 173:281-292.

Forger NG, Hodges LL, Roberts SL, Breedlove SM (1992) Regulation of motoneuron death in the spinal nucleus of the bulbocavernosus. $J$ Neurobiol 23:1192-1203.

Greengard P, Valtorta F, Czernik A, Benfati F (1993) Synaptic vesicle phosphoproteins and regulation of synaptic function. Science 259: 780-785.

Hannigan P, Kelley DB (1986) Androgen-induced alterations in vocalizations of female Xenopus laevis: modifiability and constraints. J Comp Physiol A 158:517-527.

Jordan CL, Pawson PA, Arnold AP, Grinnell AD (1992) Hormonal regulation of motor unit size and synaptic strength during synapse elimination in the rat levator ani muscle. J Neurosci 11:4447-4459.

Kelley DB (1988) Sexually dinorphic behaviors. Annu Rev Neurosci 11:225-251

Kelley DB, Dennison J (1990) The vocal motor neurons of Xenopus laevis: development of sex differences in axon number. J Neurobiol 21:869-882.

Ko C-P (1981) Electrophysiological and freeze-fracture studies of changes following denervation at frog neuromuscular junctions. J Physiol (Lond) 321:627-639.

Ko C-P (1984) Regeneration of the active zone at the frog neuromuscular junction. J Cell Biol 98:1685-1695.

Ko C-P (1985) Formation of the active zone at developing neuromuscular junctions in larval and adult bullfrogs. J Neurocytol 14:487512.

Marin ML, Tobias ML, Kelley DB (1990) Hormone-sensitive stages in the sexual differentiation of laryngeal muscle fiber number in Xenopus laevis. Development 110:703-712.

Nagaya N, Herrera A (1991) Androgens differentially affect synaptic efficacy within motor units of a sexually dimorphic muscle. Soc Neurosci Abstr 17:529.9.

Picker MD (1983) Hormonal induction of the aquatic phototactic response of Xenopus. Behavior 84:74-80.

Popov SV, Poo M-m (1993) Synaptotagmin: a calcium-sensitive inhibitor of exocytosis? Cell 73:1247 1249.

Propst JW, Herrera AA, Ko C-P (1986) A comparison of active zone structure in frog neuromuscular junctions from two fast muscles with different synaptic efficacy. J Neurocytol 15:525-534.

Pumplin DW (1983) Normal variations in presynaptic active zones of frog neuromuscular junctions. J Neurocytol 12:317-323.

Pumplin DW, Reese TS, Llinas R (1981) Are the presynaptic membrane particles the calcium channels? Proc Natl Acad Sci 78:7210 7213.

Robertson JC, Watson JT, Kelley DB (1994) Androgen directs sexual differentiation of laryngeal innervation in developing Xenopus laevis. J Neurobiol 25: in press.

Robitaille R, Charlton MP (1992) Presynaptic calcium signals and transmitter release are modulated by calcium-activated potassium channels. J Neurosci 12:297-305.

Robitaille R, Adler EM, Charlton MP (1990) Strategic location of calcium channels at transmitter release sites of frog neuromuscular synapses. Neuron 5:1453-1463.

Robitaille R, Garcia ML, Kaczorowski GJ, Charlton MP (1993) Functional colocalization of calcium and calcium-gated potassium channels in control of transmitter release. Neuron 11:645-655.

Russell W (1954) Experimental studies of the reproductive behavior 
of Xenopus laevis. I. The control mechanisms for clasping and unclasping and the specificity of hormone action. Behavior 7:113-188.

Tobias ML, Kelley DB (1987) Vocalizations of a sexually dimorphic isolated larynx: peripheral constraints on behavioral expression. J Neurosci 7:3191-3197.

Tobias ML, Kelley DB (1988) Electrophysiology and dye-coupling are sexually dimorphic characteristics of individual laryngeal muscle fibers in Xenopus laevis. J Neurosci 8:2422-2429.

Tobias ML, Bivins K, Nowicki S, Kelley DB (1991a) Amplitude modulation is an attractive feature of $X$. laevis song. Soc Neurosci Abstr 17:559.9.

Tobias ML, Marin ML, Kelley DB (1991b) Development of functional sex differences in the larynx of Xenopus laevis. Dev Biol 147:251259.

Tsien RW, Lipscombe D, Madison DV, Bley KR, Fox AP (1988) Multiple types of neuronal calcium channels and their selective modulation. Trends Neurosci 11:431-438.

Watson J, Kelley D (1992) Testicular masculinization of vocal behavior in juvenile female Xenopus laevis: prolonged sensitive period reveals component features of behavioral development. J Comp Physiol 171: 343-350.

Watson J, Robertson J, Sachdev U, Kelley DB (1993) Laryngeal muscle and motor neuron plasticity in Xenopus laevis: testicular masculinization of a developing neuromuscular system. J Neurobiol 24: $1615-1625$

Weintraub A, Bockman R, Kelley D (1985) Prostaglandin E2 induces sexual receptivity in female Xenopus laevis. Horm Behav 19:386399.

Wetzel D, Kelley D (1983) Androgen and gonadotropin control of the mate calls of male South African clawed frogs, Xenopus laevis. Horm Behav 17:388-404.

Yager D (1992) A unique sound production mechanism in the pipid anuran Xenopus laevis.. Zool J Linn Soc 104:351-375.

Zakon HH (1993) Weakly electric fish as model systems for studying long-term steroid action on neural circuits. Brain Behav Evol 42:242251 